\title{
Fixed subaortic stenosis
}

\section{Value of echocardiography for diagnosis and differentiation between various types}

\author{
F. J. TEN CATE, W. G. VAN DORP, P. G. HUGENHOLTZ, \\ AND J. ROELANDT \\ From the Department of Clinical Echocardiology, Thoraxcentre, Erasmus University, and Academic Hospital \\ 'Dijkzigt', Rotterdam, The Netherlands
}

SUMMARY The M-mode echocardiographic and cross-sectional findings in 19 patients with proven fixed subaortic stenosis are described. Based on their haemodynamic, angiocardiographic, and surgical analysis, two main groups of patients were identified. Group 1 consisted of 13 patients with the membranous form of the disease which was further subdivided into patients with a thin membrane and a normal outflow tract (group la: 3 patients) and those with a thicker fibrous ring or collar and some degree of outflow tract narrowing (group $1 \mathrm{~b}: 10$ patients).

Group 2 consisted of 6 patients with the typical long segment or tunnel form of the disease.

The outflow tract was of a normal size in all 3 patients in group 1a, and an abnormal echo, probably from the subaortic membrane, was seen in only 1 patient on $M$-mode and cross-sectional images. The narrowed left ventricular outflow tract was diagnosed with both methods in all patients in groups $1 \mathrm{~b}$ and 2. The area of narrowing extended over a longer segment in the patients in group 2, and this was more easily appreciated from the cross-sectional images.

Premature closure of the aortic valve cusps was recorded in 2 patients in group 1a, 5 in group 1b, and 2 in group 2. It seems from these findings that fixed subvalvular aortic stenosis due to an isolated discrete subaortic membrane cannot reliably be diagnosed by echo. Patients with narrowed outflow tract, however, are reliably detected by echo. The differentiation between a short and a long segment of narrowing is made with more confidence from cross-sectional images.

Fixed subaortic stenosis, a condition causing obstruction to left ventricular outflow, can be produced by an isolated discrete fibrous membrane located just beneath the aortic valve, a thicker fibrous ring or collar which is always associated with some degree of left ventricular outflow tract narrowing, or by diffuse long segment fibromuscular narrowing.

The correct differentiation between these various anatomical forms is important as they involve a different natural history. Furthermore, each type has its specific problems and a different prognosis when operation is considered for symptomatic patients (Maron et al., 1976; Katz et al., 1977). A few studies have indicated that echocardiography could be a helpful and noninvasive means for the diagnosis and the differentiation between the types

Received for publication 8 March 1978 of fixed subaortic stenosis (Davis et al., 1974; Popp et al., 1974; Roelandt, 1977). In addition, cross-sectional imaging has been proposed as an important method for the evaluation of the left ventricular outflow tract (Weyman et al., 1976).

The purpose of this study was to test both M-mode echocardiography and cross-sectional imaging for their role in diagnosing and differentiating the different types in 19 patients with proven subaortic stenosis.

\section{Subjects and methods}

Nineteen patients with fixed subaortic stenosis were included in this study. There were 8 men and 11 women (ages: 14 to 60 years: median 20). Major complaints were inappropriate exertional dyspnoea in 18, chest discomfort during exercise in 12, and dizziness, faintness, and/or syncopal attacks in 3. 
None had signs of heart failure at the time of their examination. Based on the haemodynamic, angiocardiographic, and surgical findings, patients were divided into 2 main groups (Table 1 ).

Group 1: 13 patients had the membranous form of the disease. Three had a normal left ventricular outflow tract (group 1a) whereas the other 10 had different degrees of left ventricular outflow tract narrowing over a short segment (group 1b).

Group 2: 6 patients with the long segment or tunnel type of subaortic stenosis.

HAEMODYNAMIC, ANGIOCARDIOGRAPHIC, AND SURGICAL DATA (TABLE 1)

Group 1

The diagnosis of fixed subaortic stenosis in the 13 patients of this group was mainly based on the demonstration of a significant pressure gradient between the left ventricular cavity and the left ventricular outflow tract. A distinct membrane with a subvalvular chamber beneath the aortic valve was shown in only 7 patients on the left ventricular cineangiocardiogram, in the right anterior oblique projection. In 11 patients, aortic regurgitation was seen during supra-aortic valve angiography. Nine patients were operated on. In 3, a thin subaortic membrane attached to the anterior mitral valve leaflet was found and excised; in the other 6 patients, there was a thicker membranous collar with a circumference of 2 to $5 \mathrm{~cm}$ just beneath the aortic valve, and this was excised as completely as possible. A bicuspid aortic valve was described in 1 patient and no further information on the aortic valve was available in the surgical reports. Severe concentric hypertrophy of the left ventricle was mentioned in 1 and a thickened interventricular septum in 2 patients.

Group 2

All 6 patients had a severe systolic pressure gradient across the left ventricular outflow tract. In 4 patients, the long segment narrowing of the left ventricular outflow tract was diagnosed from the left ventricular cineangiocardiogram. Aortic regurgitation was present in 4. Five patients were operated upon. Myectomy was performed in 3, myotomy in 1, and a left ventricular outflow tract reconstruction in the fifth one, who died after operation.

\section{ULTRASONIC EXAMINATION}

The apparatus used was a commercially available instrument (EchocardioVisor 03), which has both M-mode and cross-sectional imaging facilities (Roelandt, 1977). From the standard transducer position on the chest (Roelandt, 1977), several M-mode scans were made with a transducer scanning speed as constant as possible. Patients were examined in the supine or slightly left lateral recumbent position. During the examination, gain settings were continuously adjusted for best visualisation of cardiac structures. Recordings were made with a Honeywell line scan recorder (Visicorder 1856) on light sensitive paper (Kodak Linagraph, 1892). The following indices were studed on the $M$-mode recordings: width of the outflow tract at end-systole, motion of the aortic valve 
cusps, the presence of a high frequency vibration on the anterior mitral valve leaflet during diastole, and the systolic motion pattern and contraction pattern of the left ventricular walls (obliteration of left ventricular cavity during systole in the area beyond the posterior papillary muscles). Crosssectional studies were performed in the long axis sagittal plane following the left ventricular outflow tract and recorded on videotape.

During the analysis, special attention was paid to the width and the length of narrowing of the left ventricular outflow tract, the presence of left ventricular hypertrophy, and apical left ventricular cavity obliteration during systole.

Table 2 Echocardiographic findings in 19 patients with fixed subaortic stenosis

\begin{tabular}{|c|c|c|c|}
\hline Group & $1 a$ & lb & 2 \\
\hline $\begin{array}{l}\text { No. of patients } \\
\text { Narrow LVOT } 2 \mathrm{M} \text {-mode }\end{array}$ & $\begin{array}{l}3 \\
-\end{array}$ & $\begin{array}{l}10 \\
10 \\
\text { 'ridge' } \\
\text { like }\end{array}$ & $\begin{array}{l}6 \\
6 \\
\text { Long } \\
\text { segment }\end{array}$ \\
\hline $\begin{array}{l}\text { Thin 'extra' echo } \\
\text { Early closure Ao cusps } \\
\text { Flutter Ao cusps } \\
\text { AI } \\
\text { Concentric LVH } \\
\text { ASH } \\
\text { SAM } \\
\text { Apical obliteration }\end{array}$ & $\begin{array}{l}1 \\
- \\
- \\
- \\
-\end{array}$ & $\begin{array}{l}-5 \\
4 \\
5 \\
8 \\
2 \\
1 \\
5\end{array}$ & $\begin{array}{l}- \\
2 \\
3 \\
1 \\
2 \\
2 \\
1 \\
2\end{array}$ \\
\hline
\end{tabular}

Table 3 Left ventricular outflow tract width, septal and posterior wall thickness in 19 cases with fixed subaortic stenosis

\begin{tabular}{|c|c|c|c|c|}
\hline Case No. & $\underset{(\mathrm{mm})}{L V O T}$ & $\begin{array}{l}I V S \\
(\mathrm{~mm})\end{array}$ & $\begin{array}{l}L V P W \\
(\mathrm{~mm})\end{array}$ & $\begin{array}{l}\text { Ratio } \\
I V S / L V P W\end{array}$ \\
\hline 1 & 20 & 13 & 13 & $1 \cdot 0$ \\
\hline 2 & 21 & 12 & 12 & $1 \cdot 0$ \\
\hline 3 & 20 & 13 & 13 & $1 \cdot 0$ \\
\hline 4 & 17 & 18 & 12 & 1.54 \\
\hline 5 & 9 & 18 & 18 & 1.0 \\
\hline 6 & 10 & 20 & 10 & $2 \cdot 0$ \\
\hline 7 & 18 & 18 & 18 & 1.0 \\
\hline 8 & 4 & 16 & 16 & $1 \cdot 0$ \\
\hline 9 & 10 & 15 & 15 & $1 \cdot 0$ \\
\hline 10 & 17 & 15 & 15 & $1 \cdot 0$ \\
\hline 11 & 11 & 16 & 16 & 1.0 \\
\hline 12 & 10 & 18 & 18 & 1.0 \\
\hline 13 & 11 & 15 & 15 & 1.0 \\
\hline 14 & 10 & 18 & 18 & 1.0 \\
\hline 15 & 8 & 18 & 18 & 1.0 \\
\hline 16 & 10 & 14 & 14 & $1 \cdot 0$ \\
\hline 17 & 12 & 22 & 13 & $1 \cdot 6$ \\
\hline 18 & 8 & 20 & 15 & $1 \cdot 3$ \\
\hline 19 & 10 & 16 & 16 & 1.0 \\
\hline
\end{tabular}

LVOT, width in mm of left ventricular outflow tract at end-systole; IVS, interventricular septal thickness (mm) at end-diastole; LVPW, left ventricular posterior wall thickness ( $\mathrm{mm}$ ) at enddiastole.

Echocardiographic results (Tables 2 and 3)

GROUP 1 A

In only 1 of the 3 patients, was an abnormal echo seen in the left ventricular outflow tract (Fig. 1)

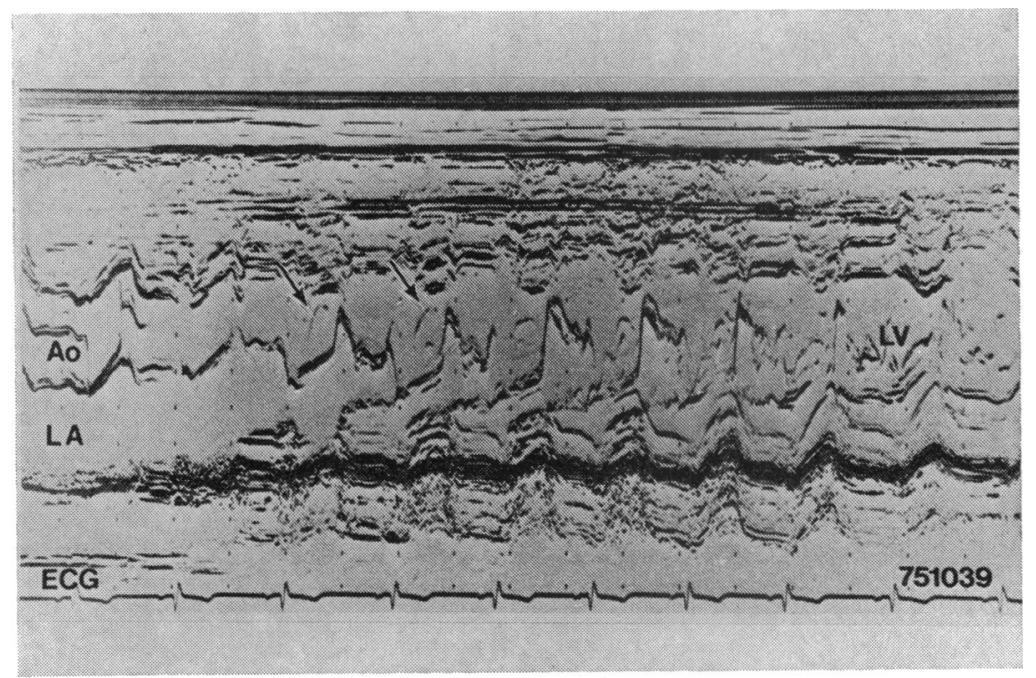

Fig. 1 Echocardiogram of a patient with fixed subaortic stenosis (group 1a). $A$ membrane was found and excised at operation. Note the normal size of the left ventricular outflow tract and the discrete echoes of membrane (see arrows). Because the membrane is attached to the mitral valve, the systolic motion pattern of that valve is abnormal. The aortic valve $(A 0)$ cusps move normally. 
and this was probably a membrane. The size of the left ventricular outflow tract was larger than 20 $\mathrm{mm}$ in all 3 patients. Premature closure of the aortic cusps was seen in 2 patients. No specific abnormalities could be detected on cross-sectional images.

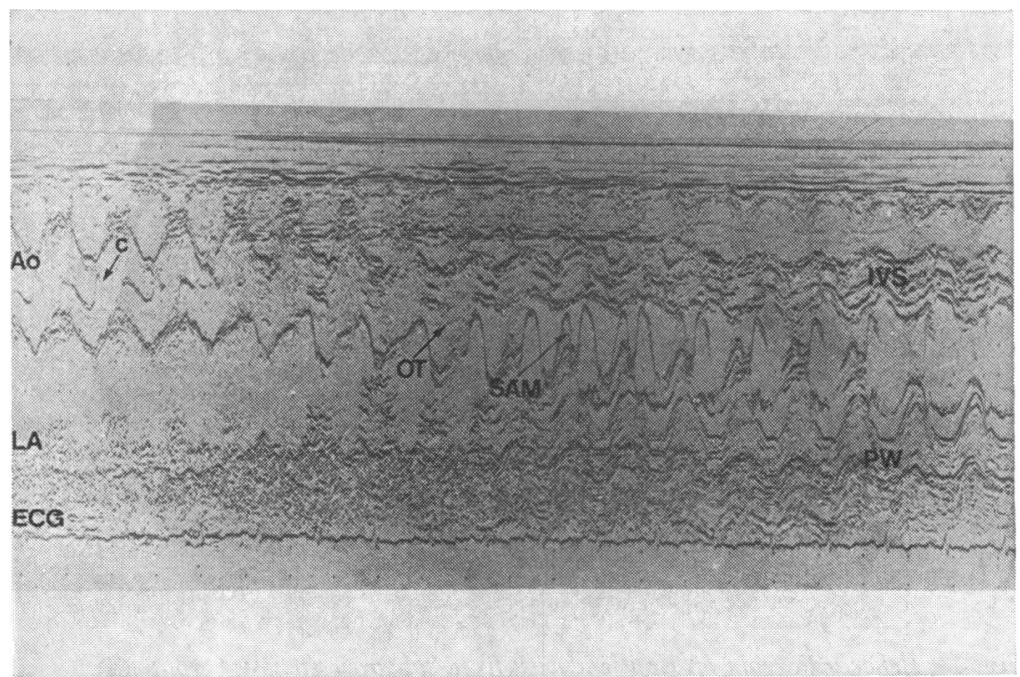

Fig. $3 M$-mode scan in a patient in group $1 b$. All features pointing to fixed subaortic stenosis are seen on this echocardiogram. In addition, the anterior mitral leaflet shows an abnormal systolic anterior motion ( $S A M$ ) (see arrow), indicative of a dynamic obstruction to left ventricular outflow. OT, outflow tract; $C$, closure. 


\section{GROUP 1B}

All 10 patients in this group had an end-systolic left ventricular outflow tract width of less than $20 \mathrm{~mm}$ (range 10 to $18 \mathrm{~mm}$; normal value $\geqslant 20 \mathrm{~mm}$ ) (Fig. 2). Five patients had an initial rapid opening of at least one cusp of the aortic valve followed by an abrupt closure shortly after the onset of ventricular ejection. In 4 of these, a distinct systolic fluttering of the aortic cusps was also recorded. A high frequency diastolic fluttering on the anterior mitral valve leaflet, suggestive of aortic regurgitation (Winsberg et al., 1970), was seen in 5 patients.
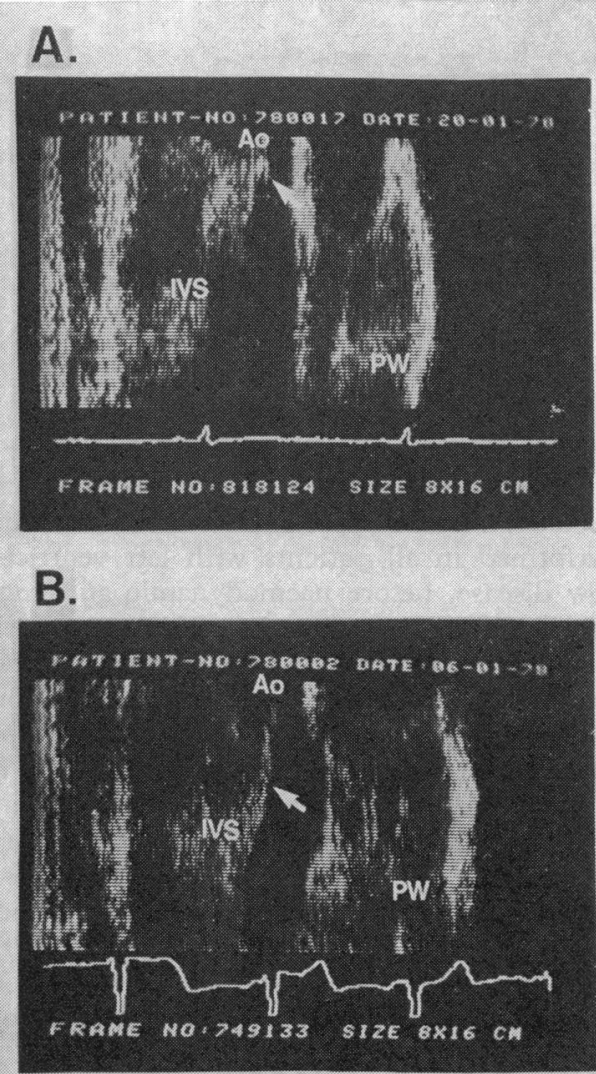

Fig. 4 (A) Ultrasonic cross-sectional image of the patient whose M-mode sector scan is described in Fig. 2. $A$ narrow left ventricular outflow tract is observed, over a short segment (see arrow), appearing as a 'ridge' just beneath the aortic valve cusps.

$(B)$ Ultrasonic cross-sectional image of the patient whose $M$-mode sector scan is described in Fig. 5. A narrow left ventricular outflow tract is observed over a long segment from a level just beneath the aortic cusps to the level of the mitral valve leaflets at left ventricular mid-cavity. For abbreviations see text.
Concentric left ventricular hypertrophy was present in 8, and 1 of them had an abnormal systolic anterior motion of the anterior mitral valve leaflet (Fig. 3). Asymmetric septal hypertrophy was present in 2 patients with end-diastolic septal thicknesses of 18 and $20 \mathrm{~mm}$, respectively. Systolic apical obliteration was seen in 5 patients. On crosssectional images, a short-segment narrowing of the left ventricular outflow tract which appeared as a 'ridge' just below the aortic valve was diagnosed in all (Fig. 4A). Severe concentric left ventricular hypertrophy was present in 8 patients, 5 of whom had systolic obliteration. In these patients, the papillary muscles were displaced more anteriorly in the left ventricular cavity. The left ventricle had an abnormal shape in 2 patients, similar to the left ventricular shape seen in patients with genetic asymmetric septal hypertrophy.

\section{GROUP 2}

All 6 patients showed a long narrow left ventricular outflow tract on their $\mathrm{M}$-mode recordings, which was quite distinctive from the echocardiographic pattern seen in patients of group $1 \mathrm{~b}$ (Fig. 5). Two patients showed an early aortic cusp closure and 3 systolic flutter. A diastolic mitral valve pattern consistent with aortic regurgitation was seen in 1 patient, and 2 had severe concentric left ventricular hypertrophy (end-diastolic septal and posterior left ventricular wall thickness $18 \mathrm{~mm}$ and $18 \mathrm{~mm}$, respectively), one of whom had systolic anterior motion. Two patients showed apical obliteration in addition to concentric left ventricular hypertrophy. Two patients had disproportionate septal thickening (septal to free posterior wall ratio $1 \cdot 3$ and 6, respectively). On their cross-sectional images, all 6 had narrowed left ventricular outflow tracts over a considerable length which extended almost to the mid-cavity level of the left ventricle (Fig. 4B). The severe concentric left ventricular hypertrophy present in 2 patients resulted in a small cavity and systolic obliteration (Fig. 6).

\section{Discussion}

Two major types of subvalvular aortic stenosis are described: the type with fixed obstruction and the type with functional obstruction to left ventricular outflow. Distinct echocardiographic abnormalities are present in the latter form (Henry et al., 1973).

The fixed form of subaortic stenosis can be divided into a membranous type, either isolated or associated with left ventricular outflow tract narrowing, and the tunnel type of subaortic stenosis. Special attention has been given in recent studies to the different prognosis and clinical 


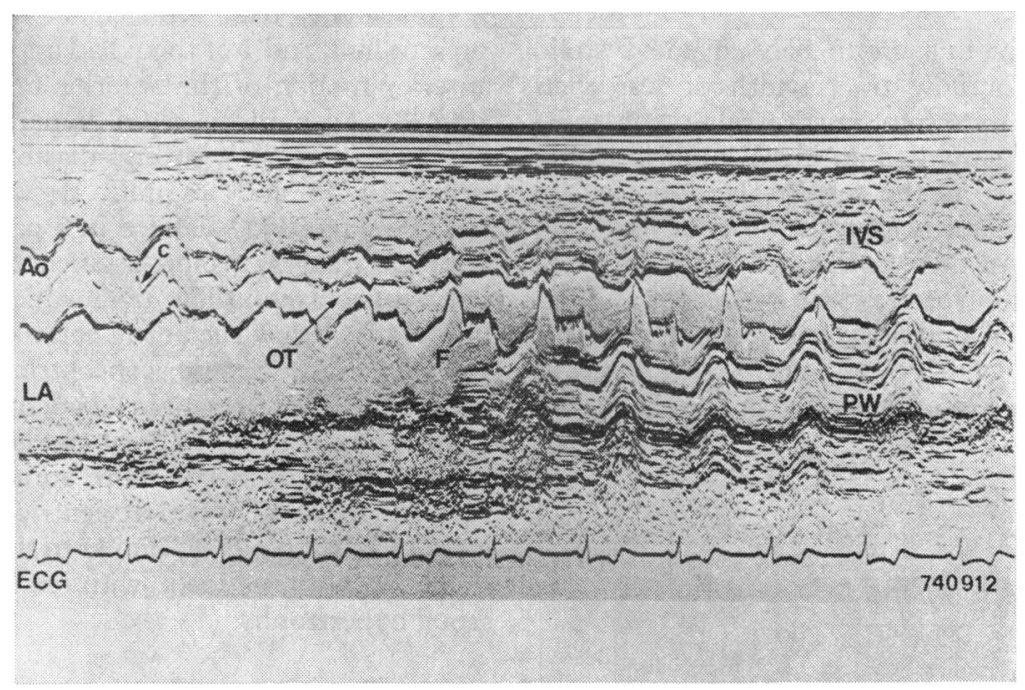

Fig. $5 M$-mode scan from the aorta $(A o)$ to the apex of the left ventricle performed with a uniform transducer speed in a patient in group 2. Note the length of the narrowed left ventricular outflow tract (OT) (see arrow). For abbreviations see text.

management for the different types of fixed subaortic stenosis. A high incidence of sudden death has been observed in the tunnel type, and surgery should not be recommended to these patients because of the risk and the lack of symptomatic improvement (Maron et al., 1976; Katz et al., 1977). However, surgical management of the membranous form is relatively safe and effective, though not always totally corrective (Kelly et al., 1972). Accurate diagnosis of the type of fixed subaortic stenosis is important. The diagnosis is often based on pressure recordings demonstrating a subvalvular gradient, since angiocardiography may fail to show the membrane or to visualise the narrowed left ventricular outflow tract. This was the case in 6 patients in our group 1 and 2 patients of group 2.

It should be recognised, however, that the quality of the angiocardiograms and especially the incidence of the $x$-ray beams are of major importance. This was not always satisfactory in our series. A positive and uniquivocal visualisation with ultrasound of an isolated subvalvular membrane is also rare, since the subaortic diaphragm was diagnosed in only 1 patient of group 1a. In a study of 11 patients, Lundström (1977) was not able to demonstrate the membrane in any of them. This is probably because the ultrasound beam is not easily aimed in a direction to hit this structure perpendicularly. With the exception of this uncommon type of the disease our findings suggest that a narrow left ventricular outflow tract can reliably be diagnosed from echo. Therefore, echocardiography may play a major role in the diagnosis and differentiation between the different types of the disease and should be performed in all patients with left ventricular outflow disease, before haemodynamic and angiographic analysis is considered. The combination of a narrow left ventricular outflow tract, early closure of the aortic cusps, the presence of aortic regurgitation and concentric ventricular hypertrophy with small cavity is suggestive if not diagnostic of the membranous form associated with narrow left ventricular outflow tract (see Fig. 2).

The tunnel form of fixed subaortic stenosis can also be missed on the cineangiocardiogram (cases 14 and 19) but was always detected by echocardiographic examination, and is best appreciated from cross-sectional images. This is because the left ventricular outflow tract can be thought of as a flattened space in these cases. The echocardiographic beam probably passes through the small dimension of the tunnel, whereas the $x$-ray beams in the anteroposterior projection are perpendicular to the tunnel, resulting in visualisation of the large dimensions of the tunnel on the angiogram (Roelandt and van Dorp, 1977).

Early systolic closure and fluttering of one of the aortic valve cusps, earlier described as characteristic of fixed subaortic stenosis (Davis et al., 1974), has not been found to be sensitive for detecting 

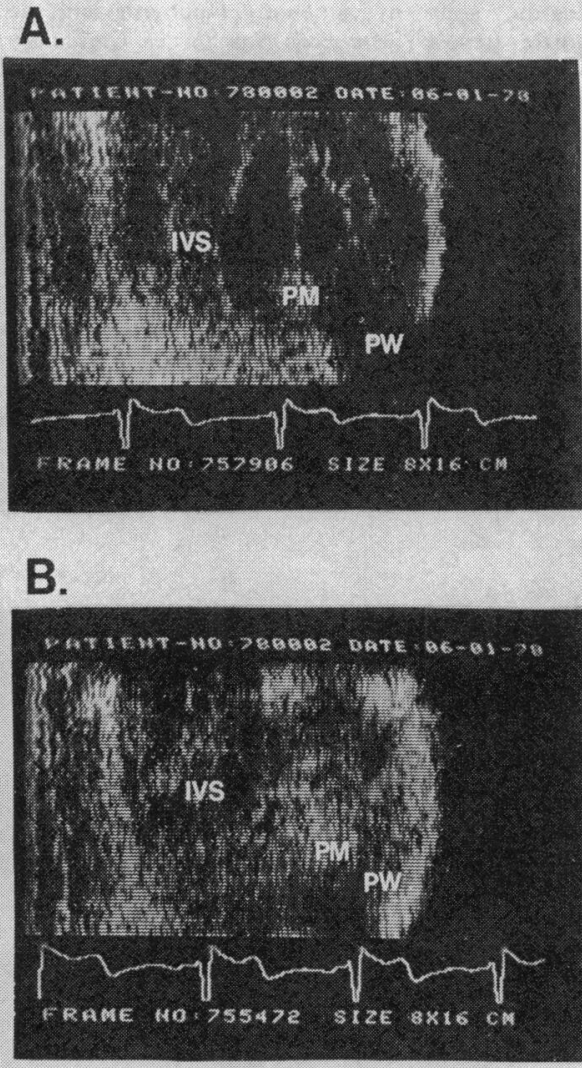

Fig. 6 (A) Ultrasonic transverse cross-sectional image at the level, just beneath the mitral leaflets at enddiastole.

The cavity of the left ventricle is well seen. The interventricular septum (IVS) and left ventricular posterior wall $(P W)$ are symmetrically hypertrophied.

(B) Same cross-section as described in Fig. $6 \mathrm{~A}$ at endsystole. Observe the systolic obliteration of the left ventricular cavity. PM, papillary muscle.

it in our series. This finding is now recognised to be a nonspecific echocardiographic abnormality (Weyman et al., 1976). There was a striking discrepancy between echocardiographic abnormalities of the mitral valve suggesting aortic regurgitation and the angiocardiographic findings. Only 6 patients were diagnosed from echo while 15 of the 19 patients had minor degrees of aortic regurgitation on their cineangiocardiograms. This discrepancy could be explained by differences in direction of the regurgitant jet. Asymmetric septal hypertrophy and an abnormal systolic anterior motion of the anterior mitral leaflet, features typically associated with idiopathic subaortic stenosis, also occur in fixed subaortic stenosis. This is important when operation is considered, and, in addition to excision of the fixed obstruction, a septal myotomy-myectomy should be performed. Otherwise, the patient may succumb in the immediate postoperative period.

The coexistence of asymmetric septal hypertrophy, systolic anterior motion of the mitral valve leaflet, and fixed subaortic stenosis suggests an interrelation between this type of subaortic stenosis and more diffuse functional types of left ventricular outflow obstruction. The history and echocardiographic study of family members of 2 patients (cases 8 and 17) showed first degree relatives with typical features of idiopathic hypertrophic subaortic stenosis, confirmed at cardiac catheterisation. A relation between the membranous form of fixed subaortic stenosis and tunnel subaortic stenosis has also been suggested (Kelly et al., 1972). This is further substantiated by the observation in one of our patients (case 14) who developed the typical form of tunnel subaortic stenosis after excision of a subaortic collar 10 years previously. Some authors believe in an age-related incidence of this disease (Katz et al., 1977). Many patients die in adolescence suddenly or as a result of intractable heart failure. However, 4 of our patients were older than 50 years, which has rarely been described.

In conclusion, it appears that echocardiography is a useful noninvasive means for the diagnosis of fixed subaortic stenosis, except for the form where there is an isolated membrane in a left ventricular outflow tract of normal size. The echo diagnosis of fixed subaortic stenosis is not always specific and may involve several investigations on $\mathrm{M}$-mode recordings and cross-sectional images. The method certainly allows a better preoperative evaluation and hence surgical management. Echocardiography will also enlarge our knowledge of the natural history of fixed subaortic stenosis.

\section{References}

Davis, R. H., Feigenbaum, H., Chang, S., Konecke, L. L., and Dillon, J. C. (1974). Echocardiographic manifestations of discrete subaortic stenosis. American fournal of Cardio$\log y, 33,277-280$.

Henry, W. L., Clark, C. E., and Epstein, S. E. (1973). Asymmetric septal hypertrophy: echocardiographic identification of the pathognomonic anatomic abnormality of IHSS. Circulation, 47, 225-233.

Katz, N. M., Buckley, M. J., and Liberthson, R. R. (1977). Discrete membranous subaortic stenosis. Report of 31 patients, review of literature, and delineation of management. Circulation, 56, 1034-1038.

Kelly, D. T., Wulfsberg, E., and Rowe, R. D. (1972). Discrete subaortic stenosis. Circulation, 46, 309-322.

Lundström, N. R. (1977). Echocardiography in the diagnosis of subaortic stenosis. In Ultrasound Echocardiography, Symposium, Lund, 1977, p. 21. Lund, Sweden. 
Maron, B. J., Redwood, D. R., Roberts, W. C., Henry, W. L., Morrow, A. G., and Epstein, S. E. (1976). Tunnel subaortic stenosis. Left ventricular outflow tract obstruction produced by fibromuscular tubular narrowing. Circulation, 54, 404-416.

Popp, R. L., Silverman, J. F., French, J. W., Stinson, E. B., and Harrison, D. C. (1974). Echocardiographic findings in discrete subvalvular aortic stenosis. Circulation, 49, 226-231.

Roelandt, J. (1977). Practical Echocardiology. Research Studies Press, Forest Grove, Oregon.

Roelandt, J., and van Dorp, W. G. (1977). Long-segment (tunnel) subaortic stenosis. Chest, 72, 222-224.
Weyman, A. E., Feigenbaum, H., Hurwitz, R. A., Girod, D A., Dillon, J. C., and Chang, S. (1976). Cross-sectional echocardiography in evaluation of patients with discrete subaortic stenosis. American fournal of Cardiology, 37, 358-365.

Winsberg, F., Gabor, G. E., and Hernberg, J. G. (1970). Fluttering of the mitral valve in aortic insufficiency. Circulation, 41, 225-229.

Requests for reprints to Dr J. Roelandt, P.O. Box 1738, The Thoraxcentre, Rotterdam, The Netherlands. 